\title{
Hydrodynamic Lubrication of Partially Textured Gas Parallel Slider Bearings with Orientation Ellipse Dimples
}

\author{
Fuxi Liu, ${ }^{1}$ Zhanlong Li $\mathbb{D}^{2}{ }^{2}$ Chunjie Yang, ${ }^{3}$ Hongbao Wu, ${ }^{1}$ Huazhu Yin, ${ }^{1}$ and Shan Jiang ${ }^{1}$ \\ ${ }^{1}$ School of Mechanical and Electrical Engineering, Hunan Applied Technology University, Changde 415100, China \\ ${ }^{2}$ School of Mechanical Engineering, Taiyuan University of Science and Technology, Taiyuan 030024, China \\ ${ }^{3}$ College of Automation, Xi'an University of Posts and Telecommunications, Xi'an 710121, China \\ Correspondence should be addressed to Zhanlong Li; lizl@tyust.edu.cn
}

Received 5 September 2021; Revised 27 October 2021; Accepted 23 November 2021; Published 18 December 2021

Academic Editor: Can Huang

Copyright $($ C 2021 Fuxi Liu et al. This is an open access article distributed under the Creative Commons Attribution License, which permits unrestricted use, distribution, and reproduction in any medium, provided the original work is properly cited.

\begin{abstract}
The hydrodynamic lubrication performance of partially textured gas parallel slider bearings with orientation ellipse dimples is investigated in this paper. By using the multigrid finite element method, the pressure distribution between a partially textured slider and a smooth slider is obtained. The geometric parameters of the ellipse dimples are optimized to maximize the average pressure under a given sliding speed. The numerical results show that geometric parameters such as orientation angle, depth, slender ratio, and area density have an important impact on hydrodynamic pressure. Besides, the effect of textured fraction on hydrodynamic pressure is investigated under a given sliding speed. It is observed that the optimum textured fraction for maximizing the average pressure is dependent on the sliding speed.
\end{abstract}

\section{Introduction}

Surface texturing is a viable option for improving the frictional performance of mechanical components. Generally speaking, the functions of surface texturing include increasing the load-carrying capacity [1], reducing the friction [2], preventing the wear particles from producing the abrasive wear [3], enhancing the lubricant retention [4], etc. With the functions surface texturing has been applied to a thrust bearing [5], piston ring [6], mechanical seal [7], and gas seal [8]. Recently, some theoretical and experimental investigations about the textured mechanical components have been carried out. Brizmer et al. [9] established a mathematical model to investigate the load-carrying capacity of a textured parallel thrust bearing. It was shown that the load-carrying capacity of a parallel thrust bearing could be improved by optimizing some parameters of the dimples. It was also demonstrated that the partially textured parallel thrust bearing have better load-carrying capacity than the fully textured parallel thrust bearing. Experimentally, Etsion et al. [5] investigated the performance of a textured parallel thrust bearing. It was found that the experimental results agree with the theoretical results. Guo et al. [10] developed a diesel engine tester to investigate the lubrication performance of a textured cylinder liner-piston ring component. They showed that the surface texture structures have an important influence on the lubrication performance of a cylinder liner-piston ring component. Pei et al. [11] investigated the performance of a textured float ring bearing. It was shown that the presence of surface texture could increase the side leakage and reduce the temperature rise. TalaIghil et al. [12] investigated the performance of a textured journal bearing. It was found that an appropriate surface texture geometry and appropriate textures distribution could improve the minimum film thickness, maximum pressure, axial oil film flow, and friction torque of a journal bearing. Yu et al. [13] investigated the performance of a textured mechanical seal. Their results showed that the presence of surface texture could reduce the temperature rise, friction torque, and friction coefficient of a mechanical seal. McNickle and Etsion [8] investigated the performance of a textured dry gas seal. It was shown that the textured gas seal runs smoother than the untextured gas seal. Fu et al. [14] investigated the hydrodynamic lubrication of a partially 
textured oil-lubricated parallel slider bearing. It was shown that the partially textured oil-lubricated parallel slider bearing have better hydrodynamic lubrication performance than the fully textured oil-lubricated parallel slider bearing.

Gas parallel slider bearings have an advantage of low friction and no pollution compared with oil-lubricated parallel slider bearings. Gas parallel slider bearings have been applied in micromaching, coordinate measuring machine, optical grinding, etc. Raeymaekers et al. [15] investigated the performance of a fully textured gas parallel slider bearing. Their results showed that the presence of surface texture could increase the average pressure. Qiu et al. [16] investigated the effect of texture shape on the load-carrying capacity of a fully textured gas parallel slider bearing. They found that the ellipsoidal dimples could provide the maximum load-carrying capacity. Subsequently, Qiu et al. [17] investigated the effect of texture shape on the friction coefficient and stiffness of a fully textured gas parallel slider bearing. Their results showed that the ellipsoidal dimples could yield the minimum friction coefficient and the highest bearing stiffness.

The effect of orientation ellipse dimples on hydrodynamic lubrication has been systematically investigated for partially textured oil-lubricated parallel slider bearings [18]. However, no investigations detailing the effect of orientation ellipse dimples on hydrodynamic lubrication seem to exist in the available literature for partially textured gas parallel slider bearings. In the present investigation, orientation ellipse dimples are conducted to evaluate the hydrodynamic lubrication of partially textured gas parallel slider bearings, and special attention is paid to the effects of orientation angle, depth, slender ratio, area density, and textured fraction on average pressure.

\section{The Model}

The schematic diagram of partially textured gas parallel slider bearings with orientation ellipse dimples is shown in Figure 1. The upper slider is partially textured and fixed. The lower slider is smooth and moves at a relative speed of $U$. The minimum film thickness between the upper slider and lower slider is $c$. The depth of each ellipse dimple is $h_{e}$.

The geometric model of the partially textured slider is shown in Figure 2. In Figure 2, the surface of the partially textured slider is divided into $n \times m\left(n=l / l_{1}\right.$ and $\left.m=l / l_{2}\right)$ imaginary rectangular cells, the ellipse dimple is located in the imaginary rectangular cell, the geometric center of the ellipse dimple is the same as the geometric center of the imaginary rectangular cell, $l$ is the length of the partially textured slider, $l_{1}$ is the length of the imaginary rectangular cell, $l_{2}$ is the width of the imaginary rectangular cell, $x$ and $y$ are the coordinates, $l_{t}$ is the length of the textured zone, $\theta$ is the orientation angle of the ellipse dimple, $a$ is half of the length of the long axis of the ellipse dimple, and $b$ is half of the length of the short axis of the ellipse dimple.

When $\theta=0^{\circ}$, the long axis of the ellipse dimple is parallel to the sliding direction. When $\theta=90^{\circ}$, the long axis of the ellipse dimple is perpendicular to the sliding direction. follows:

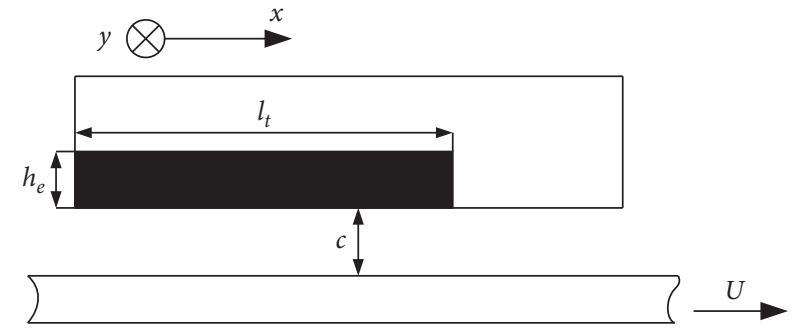

FIGURE 1: Schematic diagram of partially textured gas parallel slider bearings with orientation ellipse dimples $[14,18]$.

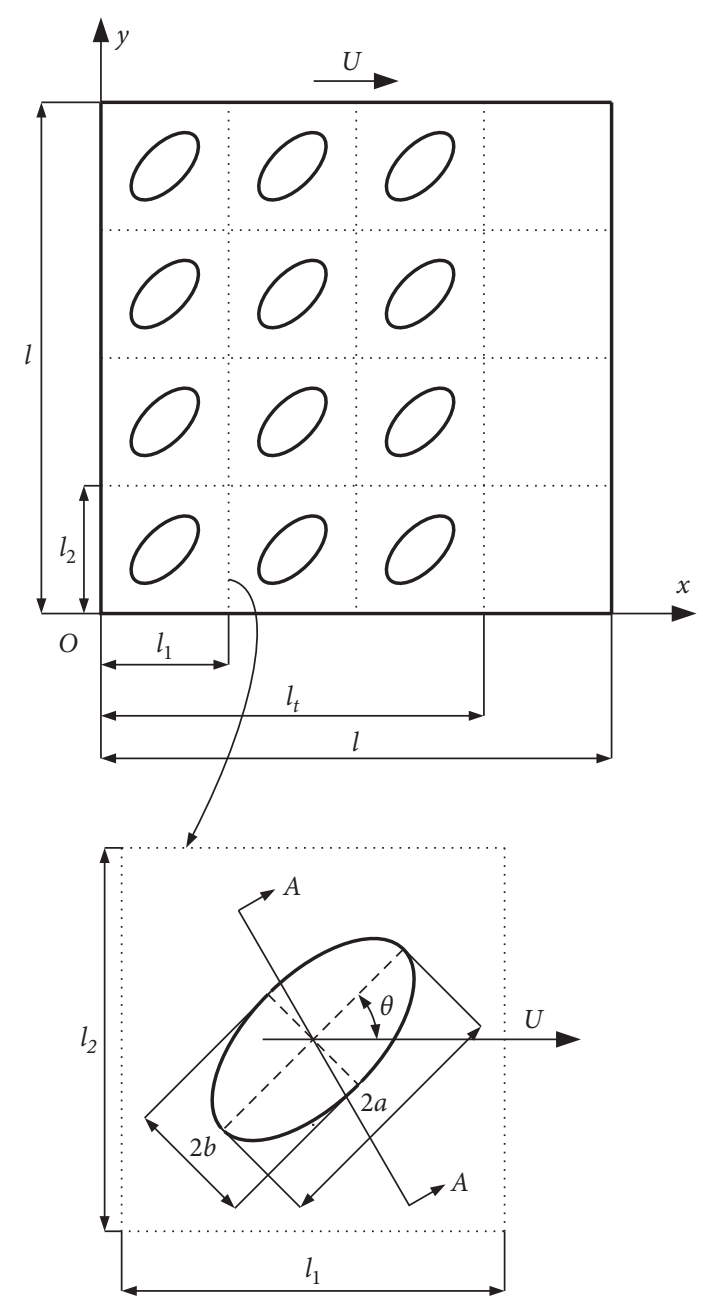

$A-A$

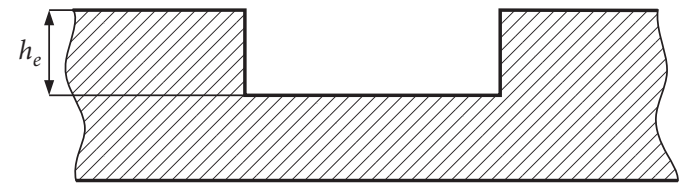

FIgURE 2: Geometric model of the partially textured slider.

$$
r_{s}=\frac{a}{b}
$$


The area density of the ellipse dimple $s_{p}$ is defined as follows:

$$
s_{p}=\frac{\pi a b}{l_{1} l_{2}} .
$$

The textured fraction $f_{t}$ is defined as follows:

$$
f_{t}=\frac{l_{t}}{l}
$$

The upper slider and lower slider are separated by the lubricant and the lubricant is the gas. It is assumed that the gas is a Newtonian fluid and the gas flow is isothermal and laminar. Hence, the steady-state Reynolds equation for partially textured gas parallel slider bearings with orientation ellipse dimples can be written as

$$
\frac{\partial}{\partial x}\left(p h^{3} \frac{\partial p}{\partial x}\right)+\frac{\partial}{\partial y}\left(p h^{3} \frac{\partial p}{\partial y}\right)=6 \mu U \frac{\partial(p h)}{\partial x},
$$

where $p$ is the gas-film pressure, $h$ is the gas-film thickness, and $\mu$ is the viscosity.

The following dimensionless variables are introduced:

$$
\begin{aligned}
X & =\frac{x}{r}, \\
Y & =\frac{y}{r}, \\
P & =\frac{p}{p_{a}}, \\
H & =\frac{h}{c},
\end{aligned}
$$

where $r=\sqrt{a b}$ is the reference value and $p_{a}$ is the ambient pressure.

The dimensionless steady-state Reynolds equation for partially textured gas parallel slider bearings with orientation ellipse dimples can be written as

$$
\frac{\partial}{\partial X}\left(P H^{3} \frac{\partial P}{\partial X}\right)+\frac{\partial}{\partial Y}\left(P H^{3} \frac{\partial P}{\partial Y}\right)=\Lambda \frac{\partial(P H)}{\partial X},
$$

where $\Lambda=\left(6 \mu U r /\left(p_{a} c^{2}\right)\right)$.

The dimensionless gas-film thickness $H$ can be obtained by

$$
\left.\begin{array}{l}
H(X, Y)= \begin{cases}1+H_{e}, & \frac{X_{1}^{2}}{a^{2}}+\frac{Y_{1}^{2}}{b^{2}} \leq 1, \\
1, & \text { elsewhere, }\end{cases} \\
X_{1}=\left(X-n_{1} L_{1}-\frac{1}{2} L_{1}\right) \cos \theta+\left(Y-n_{2} L_{2}-\frac{1}{2} L_{2}\right) \sin \theta, \\
Y_{1}=-\left(X-n_{1} L_{1}-\frac{1}{2} L_{1}\right) \sin \theta+\left(Y-n_{2} L_{2}-\frac{1}{2} L_{2}\right) \cos \theta,
\end{array}\right\}
$$

where $n_{1}=\operatorname{fix}\left(X / L_{1}\right), n_{2}=\operatorname{fix}\left(Y / L_{2}\right)$, fix is a function returning a value towards the nearest integer, $H_{e}=\left(h_{e} / c\right)$ is the dimensionless depth of the ellipse dimple, $L_{1}=\left(l_{1} / r\right)$ is the dimensionless length of the imaginary rectangular cell, and $L_{2}=\left(l_{2} / r\right)$ is the dimensionless width of the imaginary rectangular cell.

The boundary conditions of equation (6) are expressed as

$$
P(0, Y)=P(L, Y)=P(X, 0)=P(X, L)=1,
$$

where $L=(l / r)$ is the dimensionless length of the partially textured slider.

The multigrid finite element method is used to solve equation (6) and the dimensionless pressure $P$ is obtained. The solution procedure for the multigrid finite element method is that the algebraic equations formed by the finite element method are in turn smoothed by the interpolation from the coarse grid to the fine grid and the restriction from the fine grid to the coarse grid, which is the finite Newton-Raphson iteration. A multigrid W-cycle for $M=4$ is adopted in this investigation, as shown in Figure 3 . The fourth layer grid with $1025 \times 1025$ nodes, the third layer grid with $513 \times 513$ nodes, the second layer grid with $257 \times 257$ nodes, and the first layer grid with $129 \times 129$ nodes are adopted. The algebraic equations formed by the finite element method are nonlinear equations and the full approximate scheme is adopted. When the full approximate scheme is executed, the values of $v_{0}, v_{1}$, and $v_{2}$ are 12,2 , and 1 , respectively. The detailed discussion regarding the multigrid $\mathrm{W}$-cycle could be found in Wen and Huang [19]. The detailed discussion regarding the algebraic equations formed by the finite element method could be found in Liu et al. [20]. The converging condition is expressed as

$$
\left|P_{i, j}^{(1)}-P_{i, j}\right| \leq 10^{-10}
$$

where $i=1,2, \ldots, n_{x}, j=1,2, \ldots, n_{y}, n_{x}$ and $n_{y}$ are the numbers of nodes in the $x$ and $y$ direction, respectively, $P_{i, j}$ is the dimensionless pressure at the point $(i, j)$, and $P_{i, j}^{(1)}$ is the next dimensionless pressure of Newton-Raphson iteration.

After the dimensionless pressure $P$ is obtained, the dimensionless average pressure $P_{a v}$ can be obtained by

$$
P_{a v}=\frac{p_{a v}}{p_{a}}=\frac{\int_{0}^{L} \int_{0}^{L} P \mathrm{~d} X \mathrm{~d} Y}{L^{2}} .
$$

\section{Results and Discussions}

Once investigating the hydrodynamic lubrication of partially textured gas parallel slider bearings with orientation ellipse dimples, some parameters are constant: $c=2.5 \times 10^{-4} \mathrm{~mm}$, $p_{a}=0.101325 \mathrm{MPa}, \quad n=10, \quad m=10, \quad l=2.5 \mathrm{~mm}, \quad$ and $\mu=1.8 \times 10^{-5}$ Pa s.

Figure 4 shows the dimensionless gas-film pressure distributions over the partially textured slider surface with ellipse dimples in the cases of $f_{t}=0.1$ and $f_{t}=0.6$. For the two cases, the hydrodynamic pressure behaviors are similar and the hydrodynamic pressures have an optimal value at the end of the textured zone. However, the maximum dimensionless pressure in the case of $f_{t}=0.1$ is 1.16 and the 


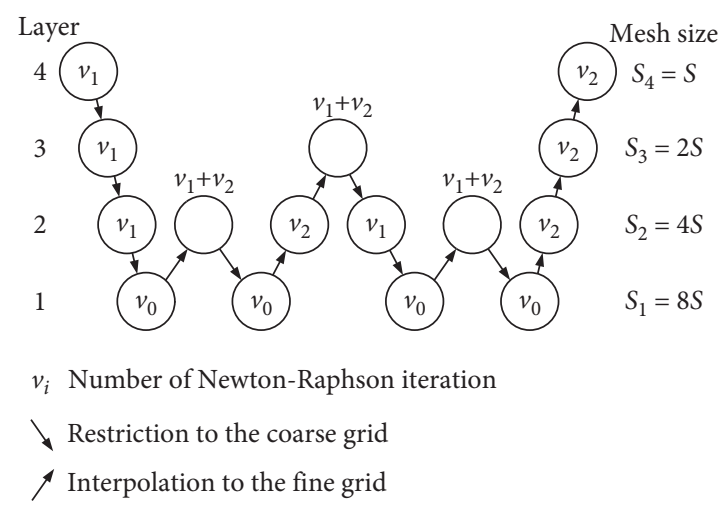

Figure 3: A multigrid W-cycle for $M=4$.

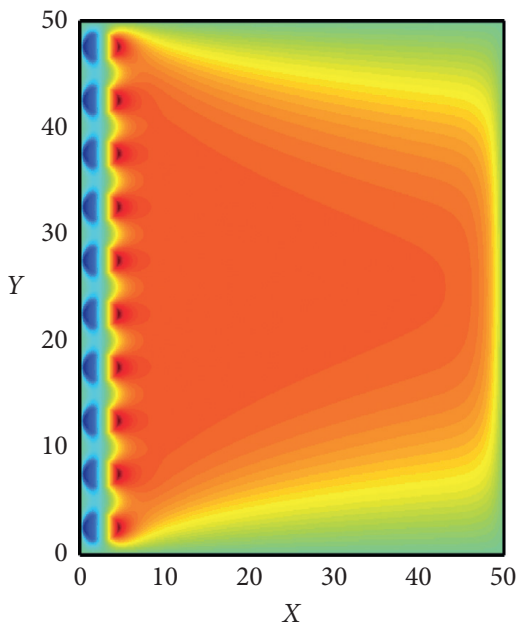

(a)

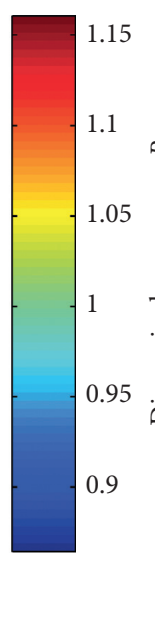

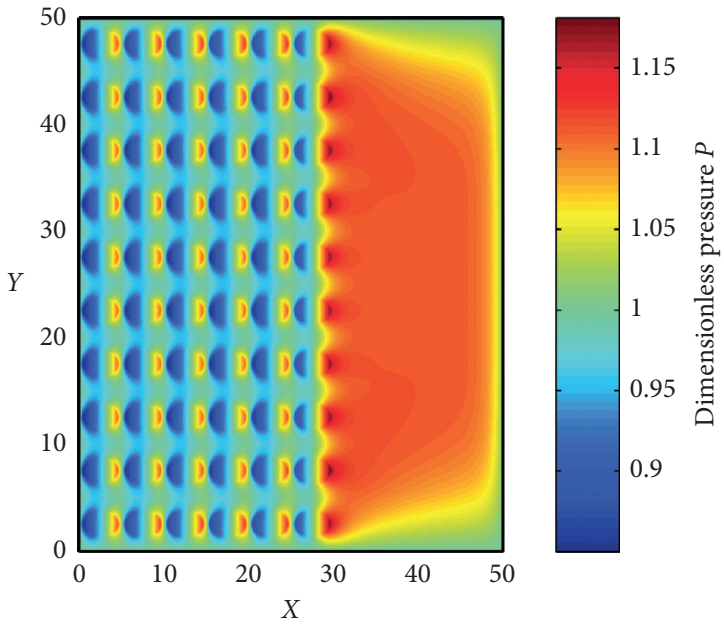

(b)

FIGURE 4: Dimensionless gas-film pressure distributions for (a) $f_{t}=0.1$ and (b) $f_{t}=0.6\left(\theta=0^{\circ}, H_{e}=1, r_{s}=1.75, s_{p}=0.3\right.$, and $\left.U=1 \mathrm{~m} / \mathrm{s}\right)$.

maximum dimensionless pressure in the case of $f_{t}=0.6$ is 1.18 .

In order to better illustrate the effect of textured fractured $f_{t}$ on hydrodynamic pressure, Figure 5 shows the dimensionless gas-film pressure distribution along the $X$ direction at $Y=27.5$. It is observed that the maximum pressure generated by each ellipse dimple could be found near the right side of each ellipse dimple and is controlled by the textured fraction. The reason for this phenomenon may be explained by the fact that the right side of each ellipse dimple is the convergence clearance and the resistance of fluid influx is controlled by the textured fraction. The results coincide with those obtained by $\mathrm{Ji}$ et al. [18].

Figure 6 shows the effect of the orientation angle of the ellipse dimple $\theta$ on dimensionless average pressure at different values of sliding speed $U$. The average pressure decreases with the increase of the orientation angle of the ellipse dimple. The results imply that the long axis of the ellipse dimple placed parallel to the sliding direction always shows the largest value of the average pressure.

Figure 7 presents the effect of the dimensionless depth of the ellipse dimple $H_{e}$ on dimensionless average pressure at different values of sliding speed $U$. With the increase of the depth of the ellipse dimple, the average pressure initially increases, reaches a maximum value, and then gradually decreases. The results imply that there is an optimum depth of the ellipse dimple to maximize the average pressure. Therefore, the depth of the ellipse dimple should be limited in order to obtain the maximum average pressure. By limiting the depth of the ellipse dimple, the pneumatic hammer vibration [21] of the gasfilm could also be efficiently avoided. Furthermore, the optimum depth of the ellipse dimple is dependent on the sliding speed.

Figure 8 shows the effect of the slender ratio of the ellipse dimple $r_{s}$ on dimensionless average pressure at different values of sliding speed $U$. The average pressure increases with the increase of the slender ratio of the ellipse dimple. Therefore, the slender ratio of the ellipse dimple should be chosen as large as possible for obtaining the maximum average pressure. The results coincide with those obtained by Ji et al. [18].

Figure 9 presents the effect of the area density of the ellipse dimple $s_{p}$ on dimensionless average pressure at different values of sliding speed $U$. The average pressure 


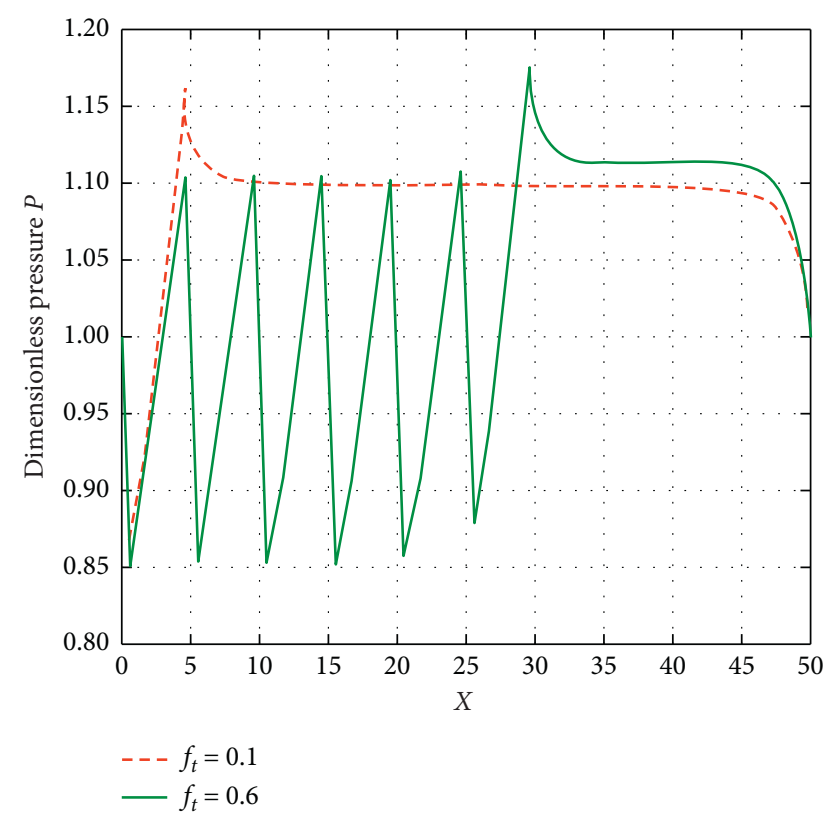

Figure 5: Dimensionless gas-film pressure distribution along the $X$ direction at $Y=27.5\left(\theta=0^{\circ}, H_{e}=1, r_{s}=1.75, s_{p}=0.3\right.$, and $U=1 \mathrm{~m} / \mathrm{s}$ ).

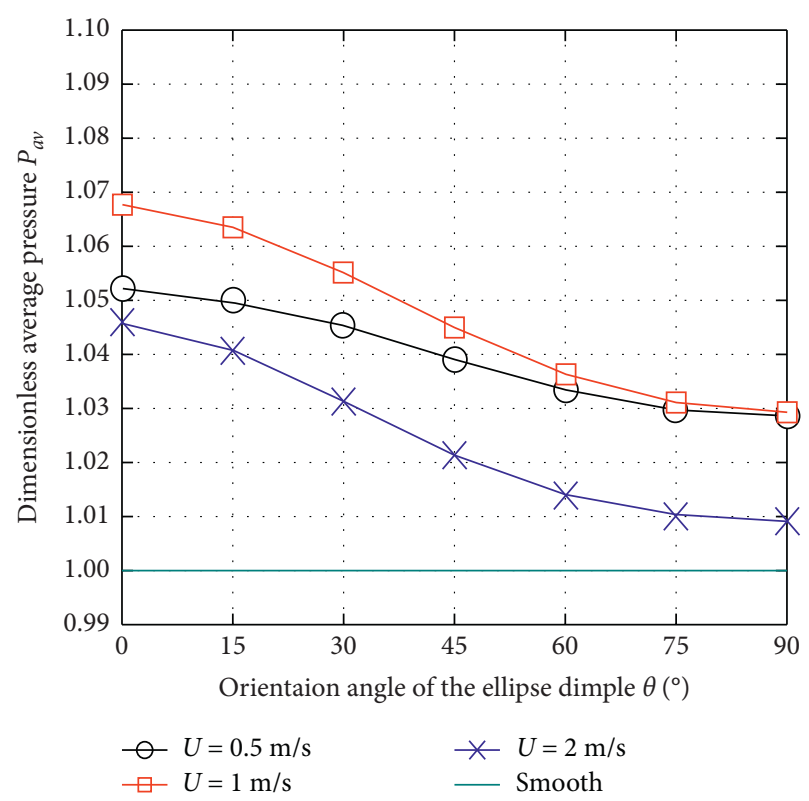

Figure 6: Dimensionless average pressure $P_{a v}$ versus orientation angle of the ellipse dimple $\theta$ at different values of sliding speed $U$ $\left(H_{e}=1, r_{s}=1.75, s_{p}=0.3\right.$, and $\left.f_{t}=0.1\right)$.

increases with the increase of the area density of the ellipse dimple. Therefore, the area density of the ellipse dimple should be fabricated as large as possible according to the practical processing techniques for obtaining the maximum average pressure. The results coincide with those obtained by Ji et al. [18].

Figure 10 shows the effect of textured fraction $f_{t}$ on dimensionless average pressure at different values of

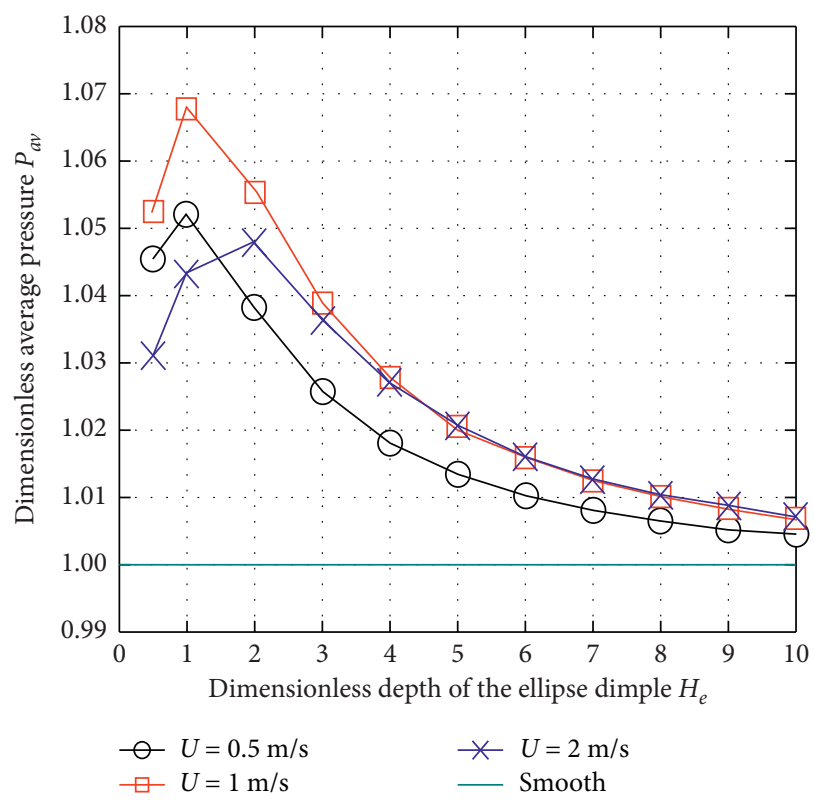

Figure 7: Dimensionless average pressure $P_{a v}$ versus dimensionless depth of the ellipse dimple $H_{e}$ at different values of sliding speed $U\left(\theta=0^{\circ}, r_{s}=1.75, s_{p}=0.3\right.$, and $\left.f_{t}=0.1\right)$.

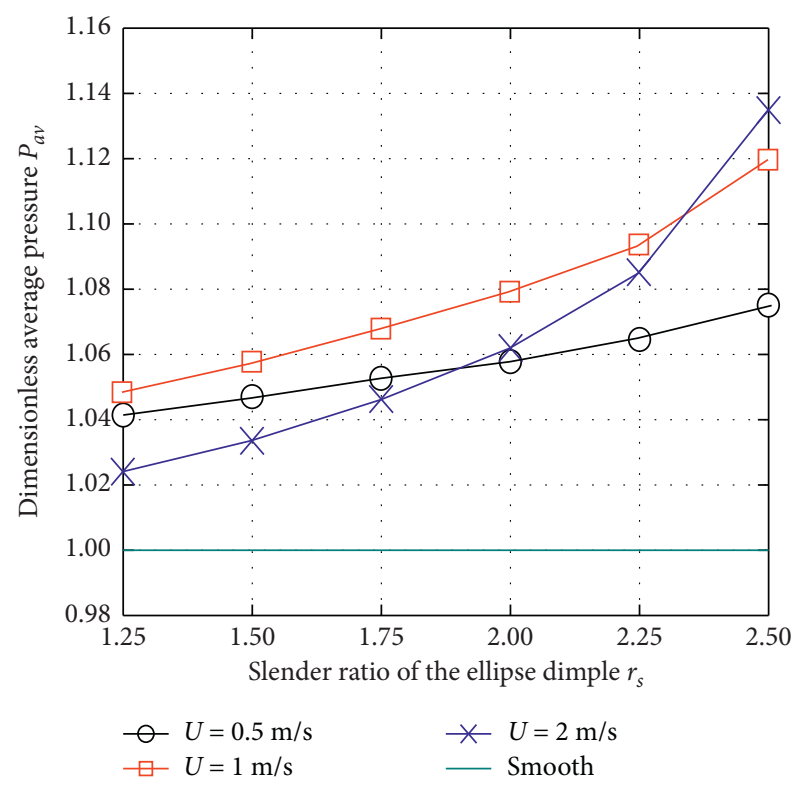

Figure 8: Dimensionless average pressure $P_{a v}$ versus slender ratio of the ellipse dimple $r_{s}$ at different values of sliding speed $U\left(\theta=0^{\circ}\right.$, $H_{e}=1, s_{p}=0.3$, and $f_{t}=0.1$ ).

sliding speed $U$. It is observed that there is an optimum textured fraction to maximize the average pressure for any given sliding speed and the optimum textured fraction is dependent on the sliding speed. It is also observed that the average pressure of partially textured gas parallel slider bearings with orientation ellipse dimples is not always lager than that of smooth gas parallel slider bearings. 


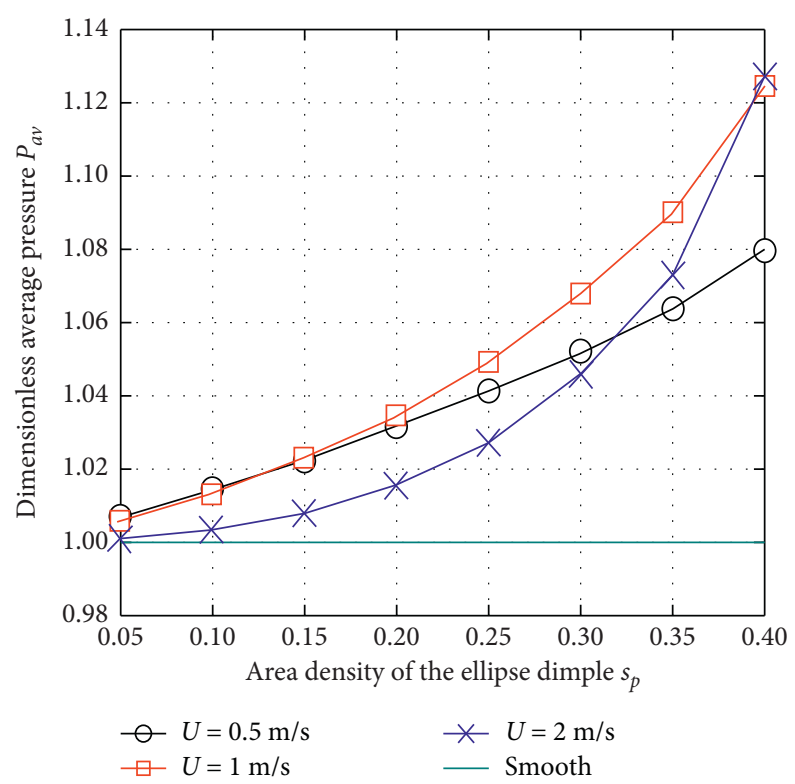

FIGURE 9: Dimensionless average pressure $P_{a v}$ versus area density of the ellipse dimple $s_{p}$ for different values of sliding speed $U$ $\left(\theta=0^{\circ}, H_{e}=1, r_{s}=1.75\right.$, and $\left.f_{t}=0.1\right)$.

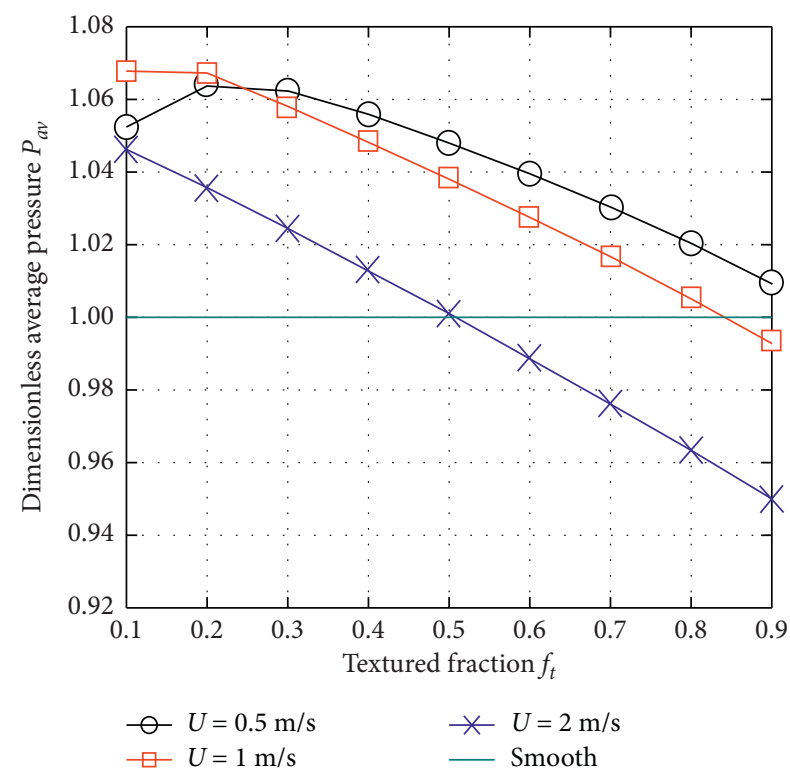

Figure 10: Dimensionless average pressure $P_{a v}$ versus textured fraction $f_{t}$ for different values of sliding speed $U\left(\theta=0^{\circ}, H_{e}=1\right.$, $r_{s}=1.75$, and $s_{p}=0.3$ ).

\section{Conclusions}

A numerical study of partially textured gas parallel slider bearings with orientation ellipse dimples is conducted. The following conclusions are obtained based on an analysis of the results:

(1) The orientation angle of the ellipse dimple has an important influence on the average pressure. The long axis of the ellipse dimple should be placed parallel to the sliding direction to obtain the maximum average pressure.

(2) The average pressure increases with the increase of the slender ratio of the ellipse dimple.

(3) The optimum depth of the ellipse dimple for maximizing the average pressure and the optimum textured fraction for maximizing the average pressure are both dependent on the sliding speed.

(4) The average pressure increases with the increase of the area density of the ellipse dimple.

\section{Data Availability}

The data used to support the findings of this study are available from the corresponding author upon request.

\section{Conflicts of Interest}

The authors declare that they have no conflicts of interest.

\section{Acknowledgments}

This study was supported by the Doctor Startup Project of Hunan Applied Technology University under Grant no. 2021 HYBS04.

\section{References}

[1] X. Wang, K. Kato, K. Adachi, and K. Aizawa, "Loads carrying capacity map for the surface texture design of $\mathrm{SiC}$ thrust bearing sliding in water," Tribology International, vol. 36, no. 3, pp. 189-197, 2003.

[2] U. Pettersson and S. Jacobson, "Influence of surface texture on boundary lubricated sliding contacts," Tribology International, vol. 36, no. 11, pp. 857-864, 2003.

[3] K. M. Li, Z. Q. Yao, Y. X. Hu, and W. B. Gu, "Friction and wear performance of laser peen textured surface under starved lubrication," Tribology International, vol. 77, pp. 97-105, 2012.

[4] I. Etsion, "Improving tribological performance of mechanical components by laser surface texturing," Tribology Letters, vol. 17, no. 4, pp. 733-737, 2004.

[5] I. Etsion, G. Halperin, V. Brizmer, and Y. Kligerman, "Experimental investigation of laser surface textured parallel thrust bearings," Tribology Letters, vol. 17, no. 2, pp. 295-300, 2004.

[6] I. Etsion and E. Sher, "Improving fuel efficiency with laser surface textured piston rings," Tribology International, vol. 42, no. 4, pp. 542-547, 2009.

[7] I. Etsion, Y. Kligerman, and G. Halperin, "Analytical and experimental investigation of laser-textured mechanical seal faces," Tribology Transactions, vol. 42, no. 3, pp. 511-516, 1999.

[8] A. D. McNickle and I. Etsion, "Near-contact laser surface textured dry gas seals," Journal of Tribology, vol. 126, no. 4, pp. 788-794, 2004.

[9] V. Brizmer, Y. Kligerman, and I. Etsion, "A laser surface textured parallel thrust bearing," Tribology Transactions, vol. 46, no. 3, pp. 397-403, 2003.

[10] Z. Guo, C. Yuan, P. Liu, Z. Peng, and X. Yan, "Study on influence of cylinder liner surface texture on lubrication performance for cylinder liner-piston ring components," Tribology Letters, vol. 51, no. 1, pp. 9-23, 2013. 
[11] S. Pei, H. Xu, M. Yun, F. Shi, and J. Hong, "Effects of surface texture on the lubrication performance of the floating ring bearing," Tribology International, vol. 102, pp. 143-153, 2016.

[12] N. Tala-Ighil, P. Maspeyrot, M. Fillon, and A. Bounif, "Effects of surface texture on journal-bearing characteristics under steady-state operating conditions," Proceedings of the Institution of Mechanical Engineers, Part J: Journal of Engineering Tribology, vol. 221, no. 6, pp. 623-633, 2007.

[13] X. Q. Yu, S. He, and R. L. Cai, "Frictional characteristics of mechanical seals with a laser-textured seal face," Journal of Materials Processing Technology, vol. 129, no. 1-3, pp. 463-466, 2002.

[14] Y. Fu, J. Ji, and Q. Bi, "The influence of partially textured slider with oriented parabolic grooves on the behavior of hydrodynamic lubrication," Tribology Transactions, vol. 55, no. 2, pp. 210-217, 2012.

[15] B. Raeymaekers, I. Etsion, and F. E. Talke, "A model for magnetic tape/guide friction reduction by laser surface texturing," Tribology Letters, vol. 28, no. 1, pp. 9-17, 2007.

[16] M. Qiu, A. Delic, and B. Raeymaekers, "The effect of texture shape on the load-carrying capacity of gas-lubricated parallel slider bearings," Tribology Letters, vol. 48, no. 3, pp. 315-327, 2012.

[17] M. Qiu, B. R. Minson, and B. Raeymaekers, "The effect of texture shape on the friction coefficient and stiffness of gaslubricated parallel slider bearings," Tribology International, vol. 67, pp. 278-288, 2013.

[18] J. Ji, Y. Fu, and Q. Bi, "The influence of partially textured slider with orientation ellipse dimples on the behavior of hydrodynamic lubrication," Industrial Lubrication and Tribology, vol. 66, no. 2, pp. 161-167, 2014.

[19] S. Z. Wen and P. Huang, Principles of Tribology, Press of Tsinghua University, Beijing, China, 2012.

[20] F. Liu, Y. Lu, Q. Zhang, Y. Zhang, P. Gupta, and N. Müller, "Load performance analysis of three-pad fixing pad aerodynamic journal bearings with parabolic grooves," Lubrication Science, vol. 28, no. 4, pp. 207-220, 2016.

[21] J. Du, G. Zhang, T. Liu, and S. To, "Improvement on load performance of externally pressurized gas journal bearings by opening pressure-equalizing grooves," Tribology International, vol. 73, pp. 156-166, 2014. 